University of Massachusetts Amherst

ScholarWorks@UMass Amherst

Chemistry Department Faculty Publication Series

Chemistry

2004

\title{
Desolvating the sample aerosol with microwave radiation: further theoretical and experimental insight into the significance of such approach
}

KO Douglass

N Fitzgerald

BJ Ingebrethsen

JE Tyson

Follow this and additional works at: https://scholarworks.umass.edu/chem_faculty_pubs

\section{Recommended Citation}

Douglass, KO; Fitzgerald, N; Ingebrethsen, BJ; and Tyson, JE, "Desolvating the sample aerosol with microwave radiation: further theoretical and experimental insight into the significance of such approach" (2004). SPECTROCHIMICA ACTA PART B-ATOMIC SPECTROSCOPY. 1028.

Retrieved from https://scholarworks.umass.edu/chem_faculty_pubs/1028 
Views and criticism

\title{
Desolvating the sample aerosol with microwave radiation: further theoretical and experimental insight into the significance of such approach
}

\author{
Kevin O. Douglass ${ }^{\mathrm{a}}$, Neil Fitzgerald ${ }^{\mathrm{b}, *}$, Bradley J. Ingebrethsen ${ }^{\mathrm{c}}$, Julian F. Tyson ${ }^{\mathrm{d}}$ \\ ${ }^{a}$ Department of Chemistry, University of Virginia, McCormick Road, P.O. Box 400319, Charlottesville, VA 22904-4319, USA \\ ${ }^{\mathrm{b}}$ Department of Chemistry and Physics, Marist College, 3399 North Road Poughkeepsie, NY 12601-1387, USA \\ ${ }^{c}$ Department of Chemistry, Medical Technology, and Physics, Monmouth University West Long Branch, NJ 07764-1898, USA \\ ${ }^{\mathrm{d} D e p a r t m e n t}$ of Chemistry, University of Massachusetts, 710 North Pleasant Street, Amherst, MA 01003-9306, USA
}

Received 26 June 2003; accepted 6 December 2003

\begin{abstract}
This study aims to determine the feasibility of using microwave energy to achieve significant improvements in sample introduction by desolvating a sample aerosol. The efficiency and mechanism of microwave desolvation were investigated. Evidence for significant interaction between aqueous droplets and microwave energy was evaluated by experimental and theoretical methods. A microwave desolvation system incorporating a Nafion dryer was evaluated for ICP-MS. A small improvement in transport to the plasma (between 51 and 60\%) was observed for magnesium, lead, and rhodium with $100 \%$ microwave power. The mechanism of desolvation was investigated using a different microwave system connected to a flame spectrometer. This system was heated, and sample introduced in the presence of a microwave field and in the absence of microwaves. No significant difference between the two cases was found indicating that the primary mechanism for increased sample transport was conductive heating of the droplets. A numerical model was developed and showed that small droplets $(5 \mu \mathrm{m})$ are incapable of absorbing sufficient energy from a $2.45 \mathrm{GHz}$ field to heat significantly due to very small absorption efficiencies as predicted by Mie theory calculations.
\end{abstract}

Keywords: Aerosol; Microwave radiation; Microwave desolvation

\section{Introduction}

In general, liquid samples are preferred in atomic spectrometric determinations due their homogeneous nature and ease of transport to the atomization source. The limitations of liquid sample introduction to a flame or plasma source are well known and have lead to the sample introduction system being described as the Achilles' heel of atomic spectroscopy [1]. Desolvation of an aqueous sample requires a significant amount of energy that can lead to significant cooling or extinction of the source. In order to overcome this restriction, liquid

\footnotetext{
*Corresponding author. Tel.: + 1-845-575-3000; fax: + 1-845-5753184.

E-mail address: neil.fitzgerald@marist.edu (N. Fitzgerald).
}

samples are usually converted into a spray and passed through a spray chamber. The spray chamber allows only a small fraction of the sample to pass to the atomization source in the form of small droplets. The inefficiency of this conventional sample introduction has lead many workers to investigate alternative sample introduction systems.

One of the most effective means to reduce solvent loading in plasma spectrometers is to desolvate the sample aerosol prior to introduction to the atomization source. This can be achieved by heating an aerosol of the sample and removing the solvent vapor produced. Desolvation systems for flame spectrometers have been reported as long ago as 1952 [2] and typically consist of a heated spray chamber connected to a condenser. 
An early example of a desolvation system used for radio frequency plasma sources was described by Veillon and Margoshes in 1968 [3]. Many similar systems have been reported for the analysis of a variety of sample matrices using plasma based instruments [4-11]. Alternative methods for the removal of solvent vapor in desolvation systems have included jet separators [12], Peltier coolers [13], and membrane dryers [14-17].

The evaporation of a liquid sample in a desolvation system typically involves heating the spray chamber walls to raise the temperature of the sample droplets. The process of heat transfer from the chamber walls to the droplets is inefficient and necessitates an applied temperature well in excess of the solvent boiling point [18]. This can cause droplets to deposit on the spray chamber walls leading to a gradual build-up of sample particles and subsequent memory effects. The use of infrared radiation as a method for evaporating solvent from an aerosol without the necessity to heat the spray chamber walls was first reported by Hell et al. in 1968 [19]. This idea was revisited by Eastgate et al. [20] who developed an infrared desolvation system and compared it to an electrically heated system of similar dimensions. The radiation system was found to improve short-term stability while sharply reducing wash out times and carry-over effects.

The application of microwave energy for the desolvation of an aqueous aerosol would appear to have significant advantages over existing systems. The irradiation of an aqueous aerosol inside a microwave transparent chamber housed in a microwave field might be expected to produce significant heating of individual droplets, leading to efficient solvent evaporation and a reduction in memory effects compared to the performance of conventional systems. The idea of microwave desolvation for flame atomic absorption spectrometry was the subject of a patent in 1982 [21]. Christiansen and Unruh [22] investigated the possibility of using microwave energy to heat an aqueous aerosol in order to develop a spray drying process for the preparation of homogeneous powders of complex metal oxide systems. The authors found little evidence for coupling of microwaves to a stream of approximately $30 \mu \mathrm{m}$ water droplets in a $2.45 \mathrm{GHz}$ microwave field of up to 2.2 $\mathrm{kW}$ power. The inefficient coupling at this frequency was suggested to be due to unfavorable dielectric and geometric effects that prevent any significant increase in droplet temperature. The authors suggest that any increase in temperature of the droplet could be reduced efficiently by the surrounding gas without significant evaporation. It was concluded that a dramatic improvement in the evaporation of small droplets may be achieved by increasing the microwave field frequency. This work is supported by the theory of remote sensing of clouds [23] and fog [24] containing water droplets of similar dimensions, which suggests that significant absorption of microwave radiation can only be achieved at frequencies significantly above $2.45 \mathrm{GHz}$ (the frequency commonly used for microwave ovens).

Gras et al. [25] developed a desolvation system incorporating a Pyrex glass spray chamber inside a domestic microwave oven operating at $2.45 \mathrm{GHz}$ and $890 \mathrm{~W}$ power and suggested that the desolvation process was due to the direct interaction between the microwave field and the droplets. The heated aerosol from this system was transported via a length of silicone rubber tubing to two condensers and the resulting material introduced to a plasma source atomic emission spectrometer. Although some differences were observed between this system and a conventionally heated desolvation system, the authors failed to prove that this was due to direct absorption by the droplets. It is possible that the materials used in this system (e.g. Pyrex glass and silicone rubber tubing) as well as deposited bulk liquid were heated by the microwave field causing thermal desolvation of the aerosol. These authors published several papers describing their microwave desolvation system [25-27] although in our opinion none of these publications conclusively proved the existence of a significant heating effect due to direct microwave interactions with aqueous droplets. In order to address some of these concerns, Canals et al. conducted experimental and theoretical studies designed to gain insight into the interaction of microwave radiation with droplets of analytical interest [28]. In this paper, the authors state that their experiments 'support the direct heating of the aerosol and, hence, the existence of an interaction between aerosol, and microwaves, in agreement with the theoretical forecast'. The authors do, however, discuss other possible contributions to the overall heating including water vapor, carrier gas and the PTFE tubing and comment that "more experiments would be necessary to specify the contribution of each of these factors that contribute to the heating of the aerosol.' Therefore, although the absorption of microwave radiation by droplets is shown, the contribution of this process to overall heating effect was not fully evaluated. Indeed, on the basis of the simple theoretical treatment used, the authors state that when heat loss processes are taken into account 'there would be no temperature increase' in the droplets due to microwave absorption. The authors also comment that the spray chamber materials have minimal contribution to the overall heating due to the low dissipation factors of PTFE and glass. While it is true that PTFE and quartz demonstrate very little heating in a microwave field, it is important to realize that common forms of borosilicate glass can heat significantly due to ionic impurities. Indeed it is quite possible to melt glass in a microwave field, a phenomenon that is well known among microwave researchers. The experiment showing a temperature increase in a microwave system in the presence of an aerosol compared to the 


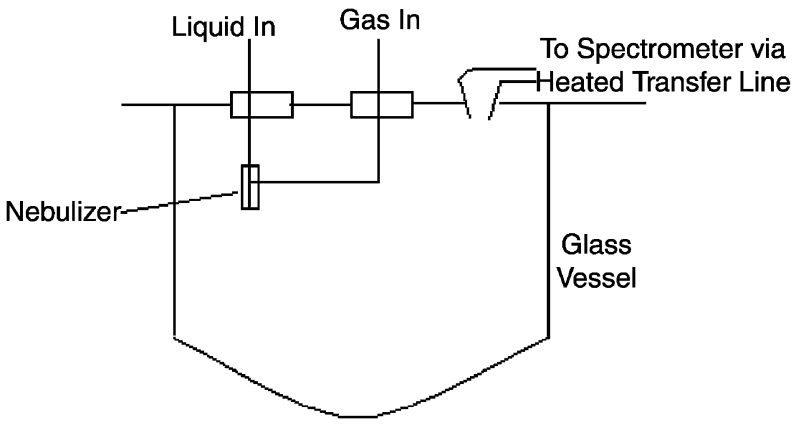

Fig. 1. Schematic diagram of the microwave test system.

effect observed with just dry gas, in our opinion, does little to address the concerns that direct interaction between droplets and microwave radiation may be an insignificant contribution to the heating process. While this system was designed to reduce the existence of bulk liquid in the microwave field, it is difficult to imagine a situation where droplets are not striking the tubing walls to some extent and possibly contributing to the overall heating effect.

In our work, the feasibility of using microwave energy in an effective desolvation system was investigated. Results from a so-called 'null' experiment, in which an identical system under similar conditions was run in the absence and presence of microwave energy, are presented. A microwave desolvation system incorporating a quartz nebulizer and spray chamber with PTFE fittings was also constructed and evaluated for ICPMS. Finally, results are presented from a numerical simulation that has been developed to describe the behavior of an aqueous droplet in a microwave field.

\section{Experimental}

\subsection{Instrumentation}

\subsubsection{Test system}

A CEM MDS-81 microwave oven with three holes of $3.5-\mathrm{cm}$ diameter in the top casing was used to test the effect of microwave energy on sample transport. It should be noted that for safety reasons any openings in the microwave casing must be 'choked' by attaching an appropriate length of metal tubing in order to prevent significant leakage of the microwaves to the surroundings. Microwave leakage was tested using a microwave survey meter (Holaday model 1501). The oven contained a 11 glass vessel (approx. $15-\mathrm{cm}$ long $\times 11-\mathrm{cm}$ diameter) fitted tightly to the top of the oven. The efficiency of sample transport was assumed to correspond to the degree of desolvation of the aerosol droplets i.e. desolvated droplets are smaller and more likely to be sweep to the detector in the nebulizer gas stream. The glass container was used as a spray chamber inside the oven in order to investigate the effect of microwave energy on an aerosol. Initial experiments were conducted using a quartz concentric nebulizer (Meinhard) placed inside the glass container (Fig. 1). The liquid stream to the nebulizer was inserted through the first of the three holes in the top of the oven through a length of $0.8 \mathrm{~mm}$ i.d. PTFE tubing. The gas stream was provided through the central hole from a compressed air cylinder at approximately $1 \mathrm{l} / \mathrm{min}$ via a length of Tygon tubing. These two holes were sealed by rubber bungs to prevent loss of the aerosol. The liquid stream to the nebulizer was delivered with a peristaltic pump (Ismatec) at 1.0 $\mathrm{ml} / \mathrm{min}$. In order to measure the exiting material in this system, a glass to metal connector was constructed in house and designed to be inserted into the third hole in the microwave oven. This allowed a heated transfer line to be firmly attached at the other end of the connector via a length of metal tubing. The heated transfer line, which was modified from a transfer line used in a gas chromatography system, consisted of a length of copper tubing (120-cm long by $1.6 \mathrm{~mm}$ i.d.) wrapped with wire which provided resistive heating contained inside an insulating sleeve. The other end of the transfer line was modified by removing the original fitting and replacing it with a length of metal tubing. The metal tube was wide enough $(3.2 \mathrm{~mm}$ o.d.) to fit tightly into the spray chamber of a flame atomic spectrometer via the hole, which originally housed the impact bead (Fig. 2). In this way, the exiting material from the transfer line was directly introduced into the instrument spray chamber. This experimental setup allowed for the measurement of the efficiency of the spray chamber system for analyte transport under various conditions. Sodium was chosen as a test element due to its strong emission intensity in an air/acetylene flame and was introduced to the sample stream by injection of $0.50 \mathrm{ml}$ of sodium chloride solution into a water carrier stream via a six port rotary valve. The spectrometer used was a IL video 22 flame spectrometer connected to an integrator (Hewlett Packard 3394A) in order to obtain a print out of the signal produced. Deionized water was used as the carrier stream. Sodium solutions were prepared by dissolving the appropriate mass of sodium chloride (Fisher Scientific) in deionized water. The temperature inside the

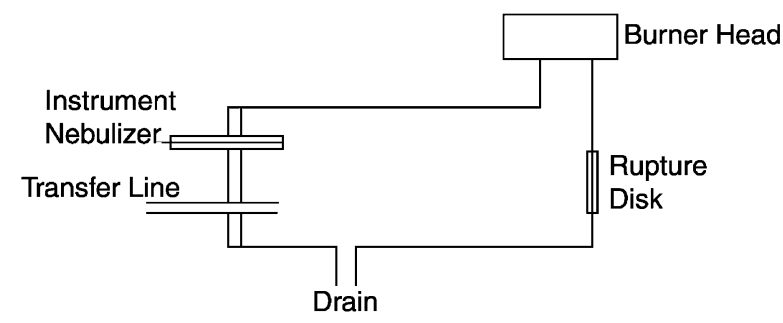

Fig. 2. Schematic diagram of the modified spectrometer sample introduction system. 


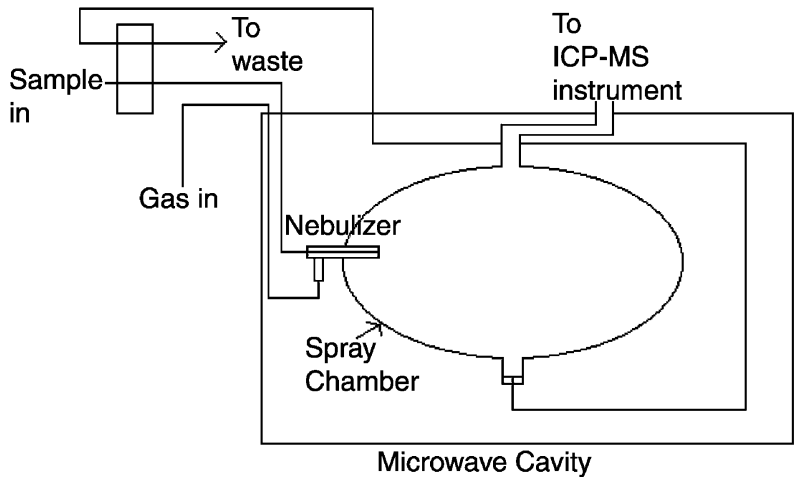

Fig. 3. Schematic diagram of the microwave heated desolvation system.

glass vessel was measured using a fibre optic probe that had been previously calibrated in a water bath against a mercury thermometer.

\subsubsection{Microwave desolvation system}

A Meinhard nebulizer attached to a glass cyclone spray chamber (Glass Expansion) was placed inside a CEM MDS-81 multi-mode microwave oven as shown in Fig. 3. The spray chamber was mounted onto a polystyrene block and placed in the centre of the cavity. Liquid was transported to the nebulizer by a peristaltic pump through $0.8 \mathrm{~mm}$ i.d. PTFE tubing, argon gas was supplied through the ICP-MS mass flow controller via a length of Tygon tubing. PTFE tubing $(0.8 \mathrm{~mm}$ i.d.) was used to pump waste from the spray chamber using a peristaltic pump. The outlet of the spray chamber was connected to the Nafion dryer device [29], operated at optimized conditions, with a length of PTFE tubing (approx. 50-cm long, $0.5 \mathrm{~cm}$ i.d.). The connecting tubing was fixed tightly into the outlet arm of the spray chamber using two O-rings to prevent gas leakage. Heating of the transfer line between the exit of the microwave oven and the Nafion dryer was accomplished using two lengths of heating tape (Barnstead, Briskheat) controlled independently by two control boxes (Geo. Ulanet Co., Robotemp). Signals were measured by ICPMS (PE Sciex 5000) with operating conditions optimized to achieve maximum signal intensities of the three test elements (Table 1).

\subsection{Procedure}

\subsubsection{Test system}

The efficiency of the system was determined by injecting $0.5 \mathrm{ml}$ of a $10 \mathrm{mg} / \mathrm{l}$ sodium solution into the spray chamber with the microwave power set at 50\% after having heated the system for 5 min at $70 \%$ power. The peak area was compared to a calibration curve for sodium constructed by injecting $0.5 \mathrm{ml}$ of $0.5,1.0,5.0$ and $10.0 \mathrm{mg} / 1$ sodium into a water carrier stream connected to the instrument nebulizer that was carried out immediately before running the microwave system. It should be noted that this calibration could be performed directly before the desolvation system was run without extinguishing the flame and that the impact bead was removed in order to accommodate the end of the transfer line. The efficiency was then calculated by determining the mass of sodium chloride entering the flame and dividing by the total mass of sodium chloride introduced into the system. In order to determine the mass of sodium entering the flame, the efficiency of the instrument nebulizer and spray chamber system must be known. This was done by introducing a known amount of sodium chloride, $0.50 \mathrm{mg}$, into the spectrometer via a flow injection manifold. The liquid in the waste line, which had previously been cleaned and filled with deionized water, was then analyzed after dilution to 50.0 $\mathrm{ml}$ with deionized water. The mean value of four replicate runs was determined and the efficiency of the sample introduction system calculated for these experimental conditions.

The temperature inside the glass vessel was measured with the fibre optic probe every $30 \mathrm{~s}$ during a run in which a sample of $0.50 \mathrm{ml}$ of $100 \mathrm{mg} / 1$ sodium was added after 2 min at 50\% microwave power and heating continued at $70 \%$ power for another $4 \mathrm{~min}$. Another set of temperature measurements were taken after heating for $2 \mathrm{~min}$ at $50 \%$ power, injection of $0.50 \mathrm{ml}$ of 100 $\mathrm{mg} / 1$ sodium into the carrier stream and then switching off the microwave power.

In order to identify the role of microwave energy in the desolvation system, a series of experiments were conducted with a sample injected into a hot spray chamber with the microwave energy on and off alternately. This was done in order to discover if the desolvation process is due to the conduction from the microwave-heated system to the droplets or by direct interaction of the microwaves with the aerosol droplets. The experiments were conducted by heating the system for $2 \mathrm{~min}$ at $70 \%$ power with a water aerosol then injecting $0.5 \mathrm{ml}$ of $100 \mathrm{mg}^{-1}$ sodium into the water stream with the power at $70 \%$ for a further $5 \mathrm{~min}$. This experiment was repeated with the power switched off after the sample was injected. In this way, the temperature of the system was similar for both experiments at the time of injection so that the degree of desolvation due to the temperature of the spray chamber should be

Table 1

Optimized ICP-MS operating conditions for microwave desolvation system

Power

Nebulizer flow rate

Plasma flow rate

Auxiliary flow rate

Sample flow rate

$995 \mathrm{~W}$
$0.898 \mathrm{l} / \mathrm{min}$
$14.51 / \mathrm{min}$
$0.9661 / \mathrm{min}$
$2.4 \mathrm{ml} / \mathrm{min}$


similar. Any increase in efficiency for the system when the microwave power was continued after the sample was injected was assumed to be due to an interaction between the microwave energy and the aerosol droplets. A statistical analysis, to determine any significant difference between the two sets of experiments was performed.

\subsection{Numerical analysis}

A theoretical numerical treatment, using the Mathcad (Mathsoft, Inc.) software program, was developed to model the processes occurring during microwave irradiation of a small aqueous droplet. The model incorporates the four most significant physical mechanisms: conduction, latent heat of vaporization, radiation absorption efficiency, and evaporation. These mechanisms were used to calculate the temperature and mass of an aqueous droplet at time increments in a looping program.

The scattering and absorption efficiency of electromagnetic radiation for a particle suspended in a medium of different refractive index is described by Mie theory. Although Rayleigh theory can be used, it is a limiting approximation suitable for situations where the particle is significantly smaller than the wavelength of the incident radiation. Mie theory relates the properties of the scattering particle shape, size, and refractive index to the angular distribution of the scattered and absorbed radiation. If the particle is absorptive, part of the light will be absorbed as heat, another part will be scattered, and the remainder will be transmitted along the incident direction. The incident beam is usually taken to be one of known intensity and wavelength, and comprised of parallel and linearly polarized components. When a plane wave is incident upon an object possessing a discrete boundary, and with optical constants different from those of the medium, a scattered wave is generated. The field vectors, which describe the electromagnetic properties of the wave in space, may be resolved into three parts, the incident wave, the wave inside the particle, and the scattered wave. These equations obey Maxwell's equations and also satisfy the vector wave equation. A particular solution of the vector wave equation is sought for which the field inside the object and the external field satisfy the various boundary conditions. Once this solution is obtained, not only is the scattered wave completely defined, but the electromagnetic conditions within the object are known as well. As a result of these solutions, the efficiency with which a particle absorbs radiation of a given wavelength as a function of radius and the real and imaginary refractive index can be calculated. For constant optical properties and incident wavelength, the absorption efficiencies can be calculated for a range of radii. The absorbed radiation flux then becomes a product of the incident radiation intensity, $I$, and the absorption effi- ciency factor, $Q_{\mathrm{abs}}$.

$q^{\prime \prime}{ }_{\mathrm{r}}=I Q_{\mathrm{abs}}$

The absorbed radiation flux and incident radiation intensity both have units of watts per area since the absorption efficiency is unit-less. In this treatment the microwave absorption efficiencies were calculated by Mie Theory using the FORTRAN codes presented by Barber and Hill [30]. Values for the microwave absorption efficiency as a function of particle size were calculated from scattering codes written specifically for this purpose. The complexity and numerical challenges involved in these calculations are discussed by Barber and Hill [30]. The resulting absorption efficiencies, $Q_{\text {abs}}$, as a function of particle size were fitted to an empirical equation and incorporated into the Mathcad program. The absorbed radiation flux for a particular particle radius can then calculated and used to determine the heat and mass loss at each time increment in the program.

Conduction may be viewed as the transfer of energy from the more energetic to the less energetic particles of a substance due to interactions between the particles. In the presence of a temperature gradient, the energy transfer by conduction must occur in the direction of decreasing temperature. Eq. (2) describes the heat flux from the particle in units of watts per square centimeter.

$q^{\prime \prime}=\frac{k\left(T_{\mathrm{s}}-T_{\mathrm{g}}\right)}{r}$

Where $T_{\mathrm{s}}$ is the temperature at the particles surface, $T_{\mathrm{g}}$ is the temperature of the gas, and $r$ is the radius of the particle. The constant $k$, the thermal conductivity, describes the ability that heat can pass through air and has units of watts per meter per Kelvin. Thus, conduction measures the heat lost per area per unit of time, where the area is the surface area of the particle.

The mechanism of mass transfer is described by evaporation. Evaporation is the reverse of growth by condensation and describes the situation when more molecules leave the surface of a particle than arrive. Mass flux is analogues to heat flux in conduction in that there exists a vapor pressure gradient instead of a temperature gradient, and gas phase molecules move in the direction of lower vapor pressure. Evaporative diffusion is the net transport of these particles in a concentration gradient. The diffusion coefficient, $D_{\mathrm{v}}$, is a property of both the diffusing molecules and the surrounding gas and relates the flux of vapor to the concentration gradient. In doing so it describes how easily the vapor can travel though the air, i.e. diffuse. As the surface vapor pressure of the diffusing species increases it creates a concentration gradient towards the 
vapor pressure of the surroundings that causes evaporation to occur. Eq. (3) describes the rate of molecules leaving the surface in terms of the droplet diameter $\left(d_{\mathrm{p}}\right)$, vapor pressure of the droplet surface $\left(P_{\mathrm{s}}\right)$ and surrounding gas $\left(P_{\mathrm{g}}\right)$, diffusion coefficient $\left(D_{\mathrm{v}}\right)$, gas constant $(R)$, and the droplet temperature $(T)$, and describes the evaporative molar flux in moles per area per time.

$m^{\prime \prime}=\frac{D_{\mathrm{v}}\left(p_{\mathrm{g}}-p_{\mathrm{s}}\right)}{r_{\mathrm{p}} R T}$

Eq. (3) assumes that the gas surrounding the particle can be treated as a continuous fluid. If the droplet diameter approaches the size of the mean free path of the gas, the rate of evaporation can increase due to the diffusing molecules 'slipping' between the gas molecules. This effect was incorporated into the model using a Fuch's Slip correction factor [31].

The last mechanism included in the analysis is heat

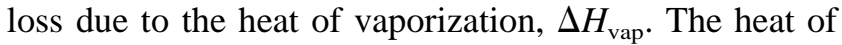
vaporization is the quantity of energy required for the liquid to vapor phase transition. The heat lost from the particle during evaporation is described by an evaporative heat flux given by

$q^{\prime \prime}{ }_{\mathrm{e}}=\Delta H_{\mathrm{vap}} m^{\prime \prime}$

Where $q^{\prime \prime}{ }_{\mathrm{e}}$ is the heat flux in watts per square centimeter, $\Delta H_{\text {vap }}$ in joules per gram is the heat of vaporization, and $m^{\prime \prime}$ is the mass flux. Thus, as water leaves the droplet it takes away a certain amount of energy, which results in a cooling of the particle.

From the heat flux from latent heat and conduction and the absorbed radiation flux, the amount of evaporation, mass flux, and resulting temperature can be determined. These mechanisms are combined in the model to predict how a particle of a certain size will heat and evaporate over a given time interval. The four mechanisms described were incorporated into Mathcad in the form of differential equations and solved numerically. The numerical solution was a 'brute-force' Euler solution in which the changing differential rates were approximated to be constant over small time increments. Numerical stability was verified by comparing results for decreasing time increments until constant values were obtained. The Mathcad program was configured to complete the specified number of steps, or loops, and output a set of variables at each time increment. The resulting data table was imported into Excel (Microsoft) for data treatment.

\section{Results and discussion}

\subsection{Test system}

The efficiency of the test system was estimated by determining the mass of sodium entering the flame and the total mass of sodium introduced into the system. By this method, the efficiency of the microwave heated system was found to be approximately $2 \%$ (based on 5 replicate measurements). This value, however, may be lower than the true value due to the increase in the amount of water vapor entering the flame in the microwave system compared to that for conventional sample introduction. This increase in water vapor may have the effect of lowering the energy of the source and decreasing the sodium emission intensity. When the system was run at room temperature, a signal could not be determined from the background noise. This suggests that desolvation is occurring in the presence of the microwave field; however, it was noted that the glass spray chamber heated significantly when a microwave field was applied. It is possible, therefore, that the heating of the spray chamber could be responsible for the desolvation effect.

In order to determine the mechanism of desolvation, an experiment was designed to compare the test system in the presence and absence of a microwave field using similar experimental conditions. The temperature of the system for the two experiments reached approximately $55{ }^{\circ} \mathrm{C}$ after 2 min when the sample was injected. The temperature of the system then increased to nearly 95 ${ }^{\circ} \mathrm{C}$ after 6 min for the experiment in the presence of the microwave field and decreased slowly to approximately $50{ }^{\circ} \mathrm{C}$ for the experiment in the absence of the microwave field. It is worth noting the temperature was measured in the center of the spray chamber where it may be cooled by the nebulizer gas and water droplets, and not at the walls of the chamber that may be at a higher temperature. The temperatures measured appear high enough to allow desolvation to occur. The heating effect may be produced by the microwaves absorption of the glass spray chamber walls, bulk water formed by the collision of droplets on the chamber walls or by direct absorption of the radiation by the water droplets. If the later mechanism were significant, a larger signal would be expected in the presence of the microwave field.

Statistical treatment of the results from a series of experiments conducted by alternating between the experiment with the microwave field switch off after the injection of the sample and left on after injection using a paired $t$-test at $95 \%$ confidence $(n=11)$ showed no significant difference between the two sets of experiments. These results suggest that desolvation in this case is occurring primarily due to conduction and that interactions between microwave radiation and aqueous droplets are not a significant contribution to the heating process.

\subsection{Microwave desolvation system}

Previous work with the test system and work reported by Gras et al. [26-28] suggested that a significant 


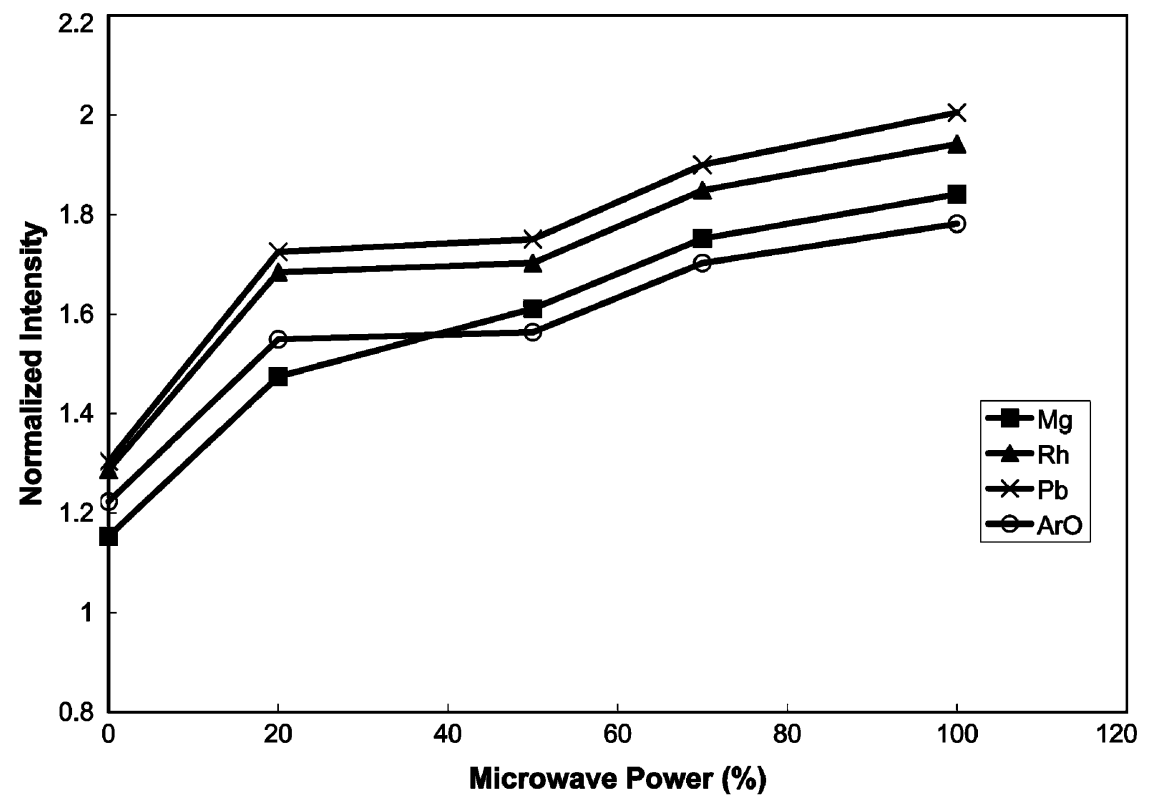

Fig. 4. Graph of normalized signal intensity against microwave power for a microwave heated desolvation system.

increase in analyte transport efficiency and more even heating may be possible using a microwave heated desolvation system. A desolvation system utilizing microwave energy and a Nafion membrane drier was constructed and tested to investigate the advantages of such a system. The system used a cyclone spray chamber in order to increase residence time of the analyte droplets in the spray chamber. The materials used inside the microwave oven (PTFE and quartz) were chosen to be virtually transparent to microwave radiation to reduce possibilities for evaporation of analyte droplets on the surfaces and subsequent memory effects. It was found to be extremely important to heat the transfer line between the spray chamber and the dryer to prevent condensation on the tubing walls. The effect of microwave power on the signal intensities produced for $\mathrm{Mg}$ 24, Pb-208, Rh-103, and ArO-56 is shown in Fig. 4 as the ratio of the signal obtained divided by the signal obtained with the same system at $0 \%$ power without heating the transfer line. It can be seen from these results that heating the transfer line without applying microwave power causes a significant increase in signal intensities. This is presumably due to desolvation occurring in the heated section of the transfer line itself. Applying microwave power has the effect of increasing signal intensities for all of the test elements. This behavior was mirrored by an increase in $\mathrm{ArO}^{+}$intensity. It is concluded that increasing the microwave power causes an increase in temperature in the spray chamber. The higher temperature in turn increases desolvation of the aerosol leading to a greater number of smaller droplets that are capable of being transported to the plasma source. The signal intensities produced, however, were lower than those obtained with the same solutions using a cross flow nebulizer and double pass spray chamber at room temperature and those obtained in the work by Gras et al. [26]. The system described by Gras et al. incorporates a single pass spray chamber that would be expected to have a higher transport efficiency than the cyclone spray chamber described here. It is worth noting that the $\mathrm{ArO}^{+}$intensity was maintained below 10000 counts $\mathrm{s}^{-1}$ in all experiments using this system due to the water removal efficiency of the Nafion drier. This is approximately an order of magnitude lower than the value typically obtained with conventional sample introduction using a double pass spray chamber and cross flow nebulizer.

\subsection{Numerical analysis}

Initial simulations were run for a range of aqueous droplet diameters irradiated at a frequency of $2.45 \mathrm{GHz}$ and intensity of $1000 \mathrm{~W} / \mathrm{cm}^{2}$. The simulation was run through several thousand steps to achieve a total simulation time of $10 \mathrm{~s}$. The graphical representations presented here (Figs. 5-7) are curve fits to the original collected data $\left(R^{2}\right.$ greater than 0.999$)$. Results are shown in Fig. 5 for three droplet diameters, 0.5, 0.1 and 0.0005 $\mathrm{cm}$. The $0.0005 \mathrm{~cm}$ droplet curve, most closely resembling the droplet size in a typical liquid sample introduction system, and $0.1 \mathrm{~cm}$ droplet curve show no significant desolvation. These results suggest that a droplet diameter of $0.5 \mathrm{~cm}$ would be necessary to achieve significant reduction in mass under these conditions. The reason for such inefficient desolvation was investigated by deactivating each of the heat flux pro- 


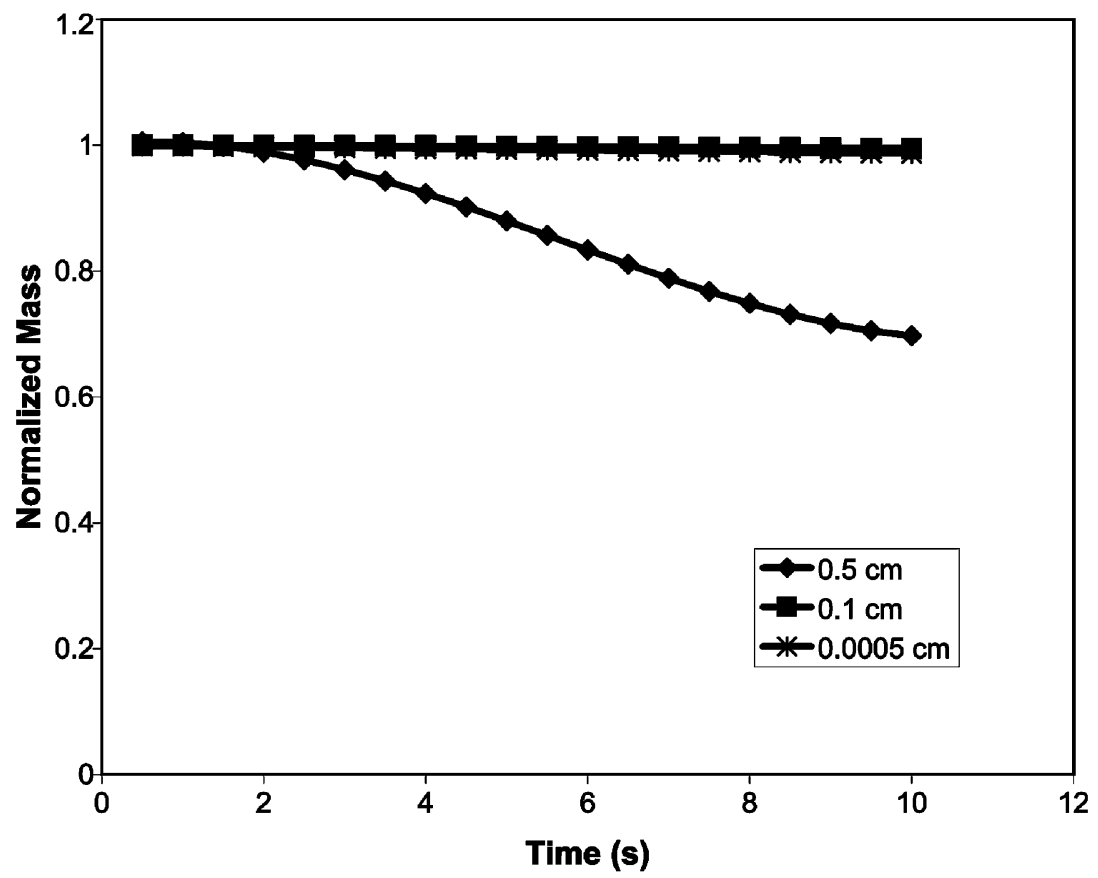

Fig. 5. Graph of normalized mass against time for aqueous droplets of varying diameters irradiated at $2.45 \mathrm{GHz}$ as calculated by numerical analysis.

cesses incorporated in the model in turn. Fig. 6 shows a plot of normalized mass against time for four different simulation runs for the irradiation of a $0.0005 \mathrm{~cm}$ droplet at $2.45 \mathrm{GHz}$. Deactivating the latent heat and conduction processes produced little effect compared to the case of all processes active. The $Q_{\text {bulk }}$ curve represents the effect of treating the droplet as bulk water, i.e. allowing radiation to be absorbed by the droplet at the same efficiency as expected for bulk samples. Our calculations confirmed the expected result that as particle diameter

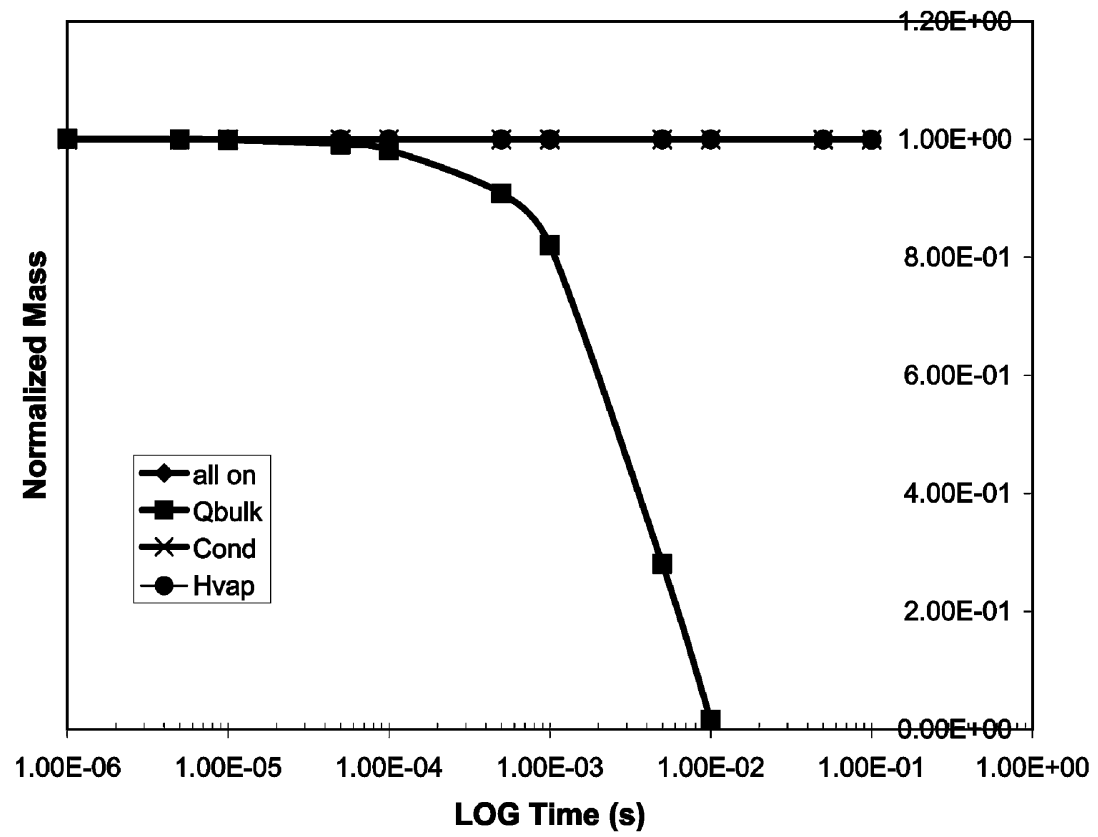

Fig. 6. Graph of normalized mass against time for an aqueous droplet irradiated at $2.45 \mathrm{GHz}$ as calculated by numerical analysis indicating the effect of deactivating heat flux processes. 'all on' indicates all processes active, ' $Q_{\text {bulk }}$ ' indicates a droplet treated as bulk water, ' $H_{\text {vap }}$ ' indicates latent heat process deactivated, ' $C_{\text {ond }}$ ' indicates conduction process deactivated. 


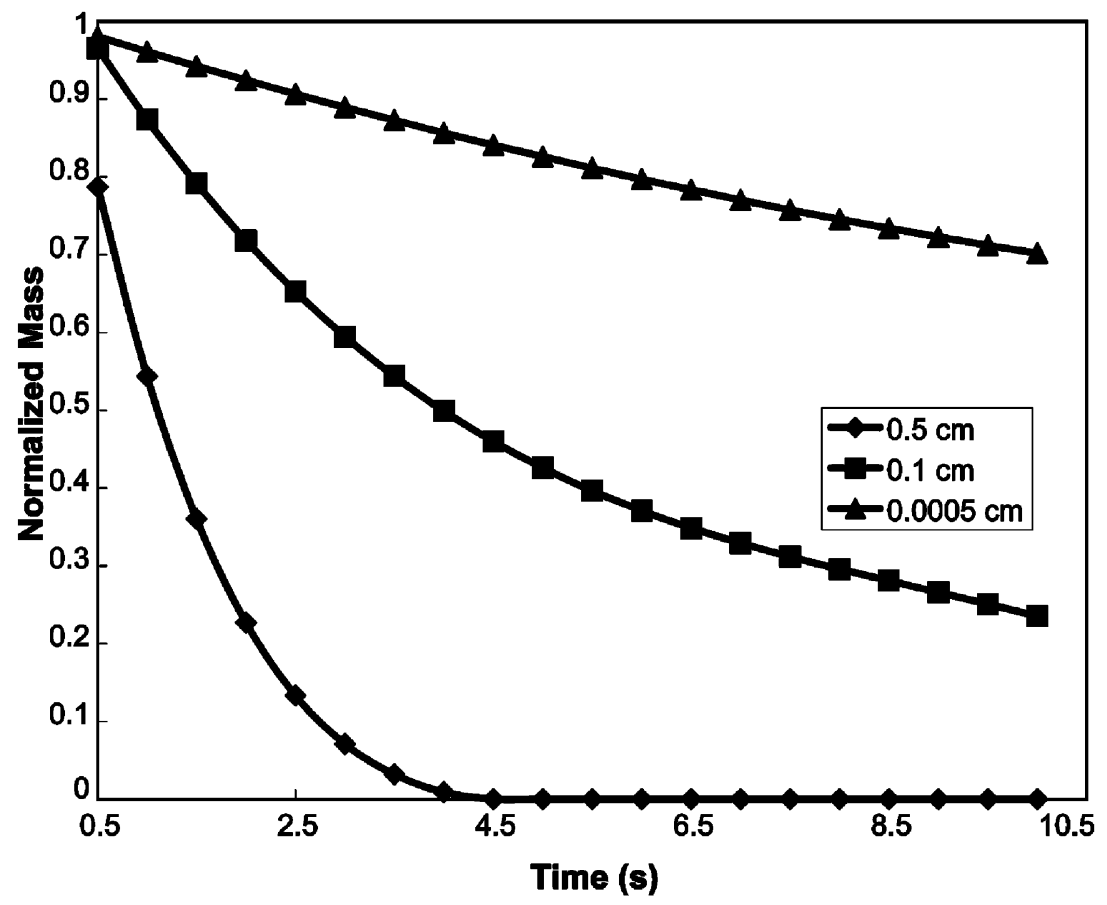

Fig. 7. Graph of normalized mass against time for droplet irradiated at $10 \mathrm{GHz}$ as calculated by numerical analysis.

gets much smaller than the wavelength of the microwaves, approximately $12.2 \mathrm{~cm}$, scattering becomes the predominant mode of interaction between the particle and the radiation and absorption becomes very inefficient. It is apparent from Fig. 6 that the inefficiency of microwave absorption at small particle size is the primary limitation to desolvation of a small aqueous droplet rather than particle cooling mechanisms. This is consistent with the droplet size predictions suggesting that absorption of microwave radiation is too inefficient to produce appreciable heating unless very large droplets are used. If large droplets are used, however, it is likely that the initial desolvation would produce smaller droplets that are less efficient at absorbing the incident radiation, therefore, effectively halting the desolvation process as heat loss mechanisms become more dominant.

The efficiency of absorption is dependent on the particle size relative to the wavelength of the incident radiation rather than the absolute particle size. An alternative approach suggested by the calculations is to increase the absorption efficiency for small droplets by decreasing the wavelength of the incident radiation. To explore this possibility a simulation was performed changing only the microwave frequency to $10 \mathrm{GHz}$ keeping all other parameters the same (Fig. 7). Although some increase in evaporation rate is predicted, significant desolvation of a small droplet does not seem feasible at this frequency. The numerical analysis results suggest some severe fundamental limitations to the feasibility of microwave desolvation and are consistent with experimental results.

\section{Conclusions}

From the experimental work described, no evidence was found for significant absorption of microwave radiation at $2.45 \mathrm{GHz}$ by small water droplets. This conclusion is supported by numerical analysis simulations, which suggest that significant absorption of microwave energy by small water droplets is unlikely to occur even at high microwave frequencies. Desolvation was found possible using a system incorporating microwave energy. The most likely mechanism for this desolvation effect is microwave heating of apparatus materials and bulk liquid inside the microwave cavity leading to conductive heating desolvation. It is our opinion that significant improvements in sample introduction systems are unlikely to occur by direct heating of aqueous droplets with microwave radiation.

\section{Acknowledgments}

The authors would like to thank CEM for financial support and Perkin-Elmer for provision of the ICP-MS instrument.

\section{References}

[1] R.F. Browner, A.W. Boom, Sample introduction: the Achilles' heel of atomic spectrometry, Anal. Chem. 56 (1984) 786A-798A. 
[2] C.A. Dubbs, Increased sensitivity for the Perkin-Elmer flame photometer - use of fine-spray hot-chamber aspiration, Anal. Chem. 24 (1952) 1654-1656.

[3] C. Veillon, M. Margoshes, A pneumatic solution nebulization system producing dry aerosol for spectroscopy, Spectrochim. Acta Part B 23 (1968) 553-555.

[4] P.D. Golden, D.H.J. Anthony, Determination of trace metals in fresh waters by inductively coupled argon plasma emission spectrometry with a heated spray chamber and desolvation, Anal. Chem. 54 (1982) 1678-1681.

[5] M.I. Marinov, Dispersity of dry aerosol, obtained from blood serum for analytical plasma spectrometry, Spectrosc. Lett. 17 (1984) 1-8.

[6] M.I. Marinov, Dry aerosol producing system for direct plasma emission analysis of body fluids, Spectrosc. Lett. 17 (1984) 33-39.

[7] M.I. Marinov, Dry aerosol producing system for direct plasma emission of body fluids, Spectrosc. Lett. 17 (1984) 151-157.

[8] H. Uchida, W.R. Masamba, T. Uchida, B.W. Smith, J.D. Winefordner, A new desolvation system for use with capacitively coupled microwave plasma emission spectrometry, Appl. Spectrosc. 43 (1989) 425-430.

[9] R. Tsukahara, M. Kubota, Studies with desolvation in inductively coupled plasma-mass spectrometry, Spectrochim. Acta Part B 45 (1990) 581-589.

[10] J.W. Lam, J.W. McLaren, Use of aerosol processing and nitrogen-argon plasmas for reduction od oxide interference in inductively coupled plasma mass spectrometry, J. Anal. At. Spectrom. 5 (1990) 419-424.

[11] N. Jakubowski, I. Feldmann, D. Stuewer, Analytical improvement of pneumatic nebulization in ICP-MS by desolvation, Spectrochim. Acta Part B 47 (1992) 107-118.

[12] A. Gustavsson, Characterization of an interface for sample introduction into an inductively coupled plasma, Spectrochim. Acta Part B 42 (1987) 111-118.

[13] S.J. Hill, J. Hartley, L. Ebdon, Determination of trace metals in volatile organic solvents using inductively coupled plasma atomic emission spectrometry and inductively couple plasma mass spectrometry, J. Anal. At. Spectrom. 7 (1992) 23-28.

[14] A. Gustavsson, O. Nygren, Characterization of a nebulizer interface for flame atomic absorption spectroscopy, Spectrochim. Acta Part B 42 (1987) 883-888.

[15] A. Gustavsson, P. Hietala, A membrane interface for aqueous sample introduction into inductively coupled plasmas, Spectrochim. Acta Part B 45 (1990) 1103-1108.

[16] A. Gustavsson, Characterization of a membrane interface for sample introduction into atom reservoirs for analytical atomic spectrometry, Spectrochim. Acta Part B 43 (1988) 917-922.
[17] K. Backstrom, A. Gustavsson, P. Hietala, A membrane interface for organic solvent sample introduction into inductively coupled plasmas, Spectrochim. Acta Part B 44 (1989) 1041-1048.

[18] B.L. Sharp, Pneumatic nebulizers and spray chambers for inductively coupled plasma spectrometry. A review. Part 2, J. Anal. At. Spectrom. 3 (1988) 939-963.

[19] A. Hell, W.F. Ulrich, N. Shifrin, J. Ramirez-Munoz, Laminar flow burner system with infrared heated spray chamber and condenser, Appl. Opt. 7 (1968) 1317-1323.

[20] A.R. Eastgate, R.C. Fry, G.H. Gower, Radiation vs. conduction in heated spray chamber desolvation for inductively coupled plasmas, J. Anal. At. Spectrom. 8 (1993) 305-308.

[21] H. Berndt, Patent No DE3026155A1, Germany, 1982.

[22] D.E. Christiansen, W.P. Unruh, Use of a $\mathrm{TM}_{010}$ microwave cavity at $2.45 \mathrm{GHz}$ for aerosol and filament drying, Ceram. Trans. 21 (1991) 597-604.

[23] L. Tsang, J. Kong, R. Shin, Theory of Microwave Remote Sensing, J. Wiley and Sons, New York, 1985.

[24] R.E. Collin, Antennas and Radiowave Propagation, McGrawHill, New York, 1985.

[25] L. Gras, J. Mora, T.L. Todoli, V. Hernandis, A. Canals, Behaviour of a desolvation system based on microwave radiation heating for use in inductively coupled plasma atomic emission spectrometry, Spectrochim. Acta Part B 52 (1997) 1201-1213.

[26] J. Mora, A. Canals, V. Hernandis, E.H. van Veen, M.T.C. de Loos-Vollebregt, Evaluation of a microwave desolvation system in inductively couple plasma mass spectrometry with low acid concentration solutions, J. Anal. At. Spectrom. 13 (1998) 175-181.

[27] L. Gras, J. Mora, J.L. Todoli, A. Canals, V. Hernandis, Microwave desolvation for acid sample introduction in inductively coupled plasma atomic emission spectrometry, Spectrochim. Acta Part B 54 (1999) 469-480.

[28] A. Canals, L. Gras, J. Mora, V. Hernandis, J. Margineda, M. Rojo, J. Munoz, Insight into the interaction of the microwave radiation with droplets of interest in analytical chemistry, Spectrochim. Acta Part B 54 (1999) 333-342.

[29] N. Fitzgerald, J.F. Tyson, Reduction of water loading effects in inductively coupled plasma mass spectrometry by a Nafion membrane dryer device, J. Anal. At. Spectrom. 13 (1998) 13-16.

[30] P.W. Barber, S.C. Hill, Light Scattering by Particles: Computational Methods, World Scientific, New Jersey, 1990.

[31] W.C. Hinds, Aerosol Technology - Properties, Behavior and Measurement, John Wiley and Sons, New York, 1982. 